\title{
Working
}

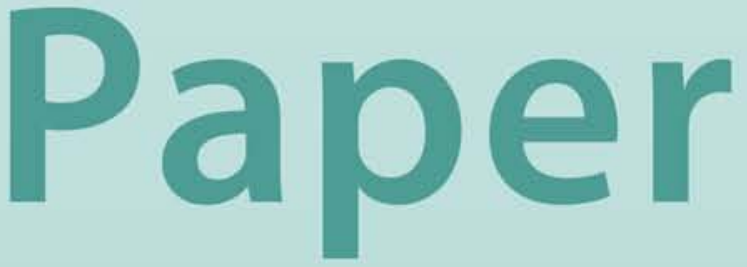


Alternative Models of Wage Dispersion

Damien Gaumont, Martin Schindler, and Randall Wright 


\title{
IMF Working Paper
}

Research Department

\section{Alternative Models of Wage Dispersion}

\author{
Prepared by Damien Gaumont, Martin Schindler and Randall Wright ${ }^{1}$ \\ Authorized for distribution by Eswar Prasad
}

March 2005

\begin{abstract}

\section{This Working Paper should not be reported as representing the views of the IMF.} The views expressed in this Working Paper are those of the author(s) and do not necessarily represent those of the IMF or IMF policy. Working Papers describe research in progress by the author(s) and are published to elicit comments and to further debate.
\end{abstract}

We analyze labor market models where the law of one price does not hold-that is, models with equilibrium wage dispersion. We begin by assuming workers are ex ante heterogeneous, and highlight a flaw with this approach: if search is costly, the market shuts down. We then assume workers are homogeneous, but matches are ex post heterogeneous. This model is robust to search costs, and it delivers equilibrium wage dispersion. However, we prove the law of two prices holds: generically, we cannot get more than two wages. We explore several other models, including one combining ex ante and ex post heterogeneity, which is robust and can deliver more than two-point wage distributions.

JEL Classification Numbers: D83, J31, J63

Keywords: Search equilibrium, wage posting, wage dispersion, labor theory

Author(s) E-Mail Address: damien.gaumont@free.fr; mschindler@imf.org; rwright@econ.upenn.edu

\footnotetext{
${ }^{1}$ Damien Gaumont is a Professor of Economics at the Université Panthéon-Assas (Paris II), Martin Schindler is an Economist in the Financial Studies Division of the IMF Research Department, and Randall Wright is a Professor of Economics at the University of Pennsylvania. The authors thank Dale Mortensen, Jim Albrecht, Ken Burdett, and Ken Wolpin for their input, and the Federal Reserve Bank of Cleveland, ERMES at Paris II, and the National Science Foundation for research support.
} 


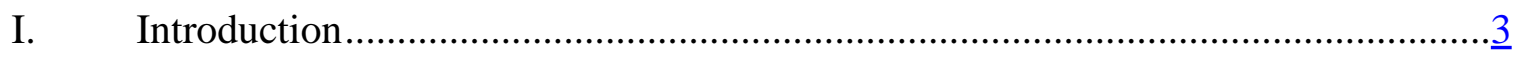

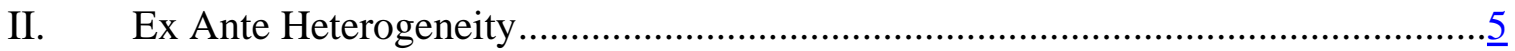

A. A Simple Model .......................................................................................

B. Alternative Assumptions ......................................................................... $\frac{9}{9}$

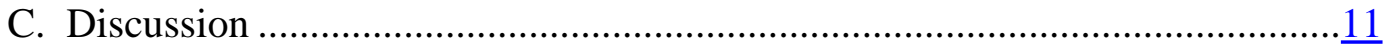

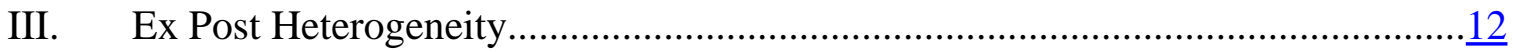

A. Permanent Shocks .............................................................................. 12

B. The Law of Two Wages ......................................................................

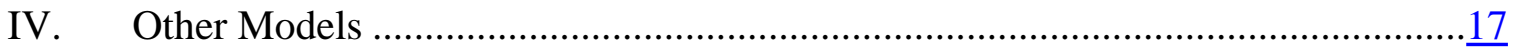

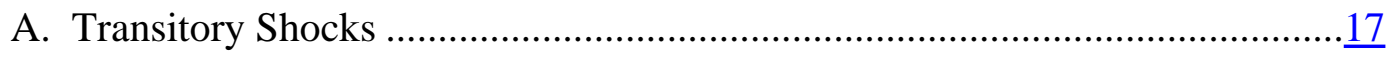

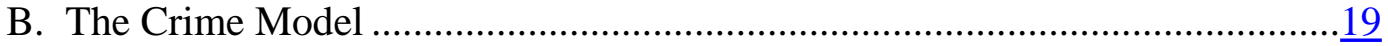

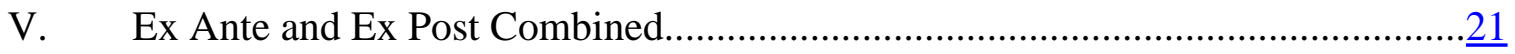

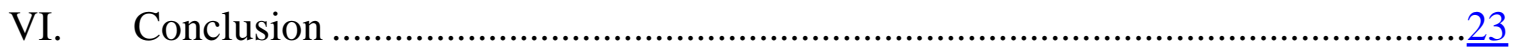

Figures

1. Multiple Equilibria.................................................................................

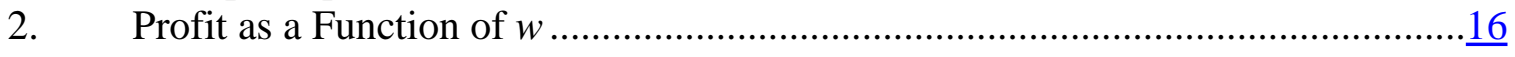

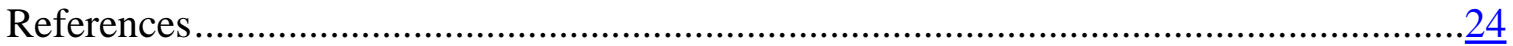




\section{INTRODUCTION}

According to Mortensen (2003, p. 9), "If the law of one price were to hold in the labor market, similar workers would not be paid differently.” This observation is both obvious and deep. The fact is, similar workers do appear to be paid differently. As Mortensen (2003, p.1) reports, although hundreds if not thousands of empirical studies that estimate so-called human capital wage equations verify that worker characteristics that one could view as indicators of labor productivity are positively related to wages earned, the theory is woefully incomplete in its explanatory power. Observable worker characteristics that are supposed to account for productivity differences typically explain no more than 30 percent of the variation in compensation. What explains the rest? It is clear that one needs a model with some sort of frictions to address the issue.

Search theory is ideally suited to the task. In their survey, Eckstein and van den Berg (2005, p. 25) argue that "equilibrium search models provide a framework to empirically analyze the sources of wage dispersion: (a) workers heterogeneity (observed and unobserved); (b) firm productivity heterogeneity (observed and unobserved); and (c) market frictions. The equilibrium framework can ... "empirically measure the quantitative importance of each source.” For example, van den Berg and Ridder (1998) estimate that up to 25 percent of wage variability is attributable to frictions, in the sense that this is what would emerge from a model without assuming heterogeneity. Postel-Vinay and Robin (2002) estimate up to 50 percent.

It is perhaps fair to say that the benchmark model for studying wage dispersion is the one developed by Burdett and Mortensen (1998), which is based on wage posting and on-the-job search. The goal of this paper is to explore some alternative models where the law of one price does not hold in the labor market. It is not that there is anything especially wrong with Burdett-Mortensen. ${ }^{2}$ However, it is good to have some alternatives on the table. These alternatives need not be mutually exclusive, of course; there may be several reasons for wage dispersion in the world, and it seems interesting to consider various options, perhaps ultimately integrating the different models in one framework and using the data to measure the importance of each. Here we are not that ambitious, and the goal is to develop theoretically several alternative models that each imply the possibility of a nondegenerate wage distribution.

We emphasize that it is not easy to get wage dispersion across homogeneous workers in equilibrium, in the sense that the well-known Diamond (1972) model, which seems on the surface the natural model in which to think about the issues, predicts a single wage even in the presence of search frictions. Of course, there are several well-known ways to get around

\footnotetext{
${ }^{2}$ There are some issues with this model, however, such as the fact that the baseline model predicts an unrealistic wage distribution, in the sense that the density is upward sloping (there are more high wage than low wage workers). This can be fixed by adding firm heterogeneity or ex ante firm investments, for example, as discussed in Mortensen (2000, 2003), but it would be nice if a simpler version was more in line with the data.
} 
this result, including: Burdett and Mortensen (1998), which introduces on-the-job search; Burdett and Judd (1983), which introduces the idea that some workers may get multiple offers; and Albrecht and Axell (1984), which introduces heterogeneous outside options for workers (values of leisure). Note that in all of these examples there is a sense in which workers are heterogeneous, but the point is that they have the same productivity and yet still can end up receiving different wages in equilibrium. In any case, the goal here is to explore some new models and ideas. ${ }^{3}$

We begin with models where workers have ex ante heterogeneous outside options, in the spirit of Albrecht and Axell (1984), although the details in the version we present are quite different. We point out a flaw in this class of models: once we introduce positive costs of search, no matter how small, equilibrium unravels. Thus, there will always be one type who will drop out, but once they do, another type drops out, and so on, until we are back to Diamond (1971). Given this, we introduce a framework where workers are homogeneous but matches are heterogeneous. That is, ex ante all workers are the same, but there are matchspecific shocks so that different workers attach different valuations to different jobs (although productivity is still the same). In this model the market does not shut down for positive search costs, and it does deliver equilibria where the law of one price fails. However, we prove that the law of two prices holds: as shown in Curtis and Wright (2004) in the context of monetary theory, in search models with match-specific shocks one can get more than one price, but generically one cannot get more than two.

We also explore some other models. To motivate these, note that in the Albrecht-Axell or Burdett-Judd style models, the reason different firms may post different wages is that highwage firms have a high inflow of workers (they recruit faster). In Burdett-Mortensen, highwage firms also have a high inflow of workers and additionally have a low outflow (they lose workers more slowly). For completeness, we present a model where all firms have the same inflow but high-wage firms have a low outflow, and one where high-wage firms get better performance from their workforce. ${ }^{4}$ In each case we prove that the law of one price does not hold, and that the law of two prices does. We also show how to combine approaches. This is important because the unraveling of models with search costs and the law of two prices can both be overturned when we have both ex ante and ex post shocks. Hence, the combination of both delivers a robust model with an empirically more interesting wage distribution.

\footnotetext{
${ }^{3}$ In this paper we are interested in models where firms post wages, as opposed to bargaining with individual workers after they meet. Any bargaining model with heterogeneous agents or heterogeneous matches, such as Mortensen and Pissarides (1994), can generate wage dispersion, but we follow the literature that asks how one can get a nondegenerate distribution of posted wages.

${ }^{4}$ The first of these is in the spirit of the Burdett, Lagos, and Wright (2003) model of crime, although considerably simpler; the second is in the spirit of the efficiency wage literature, such as Shapiro and Stiglitz (1984), although as far as we know, existing efficiency wage models do not generate endogenous wage dispersion.
} 
The rest of the paper is organized as follows. In Section II, we present some models with ex ante heterogeneity and discuss unraveling. In Section III, we introduce models with ex post heterogeneity and prove the law of two wages. In Section IV, we discuss some other approaches. In Section V, we combine ex ante and ex post heterogeneity. In Section VI we conclude.

\section{EX ANTE HETEROGENEITY}

\section{A. A Simple Model}

There is a $[0,1]$ continuum of firms and a $[0, L]$ continuum of workers. There are $K$ types of workers. A measure $L_{j}$ of workers are type $j$, and they have utility of leisure $b_{j}$ where we order types such that $b_{j+1}>b_{j}, j=1, \ldots, K$, and $\sum_{j} L_{j}=1$. Firms post wages. Each firm has a constant returns technology with labor as the only input and productivity $y>b_{K}$ (if there are any workers with $b_{j}>y$, they will never be hired and thus drop out). For now we follow Burdett and Mortensen (1998) and assume firms are interested in maximizing steady state profit and will hire as many workers as are willing to accept; we consider different models of firm behavior below. All agents are risk neutral and discount at rate $r$. Unemployed workers contact firms at rate $\alpha_{w}$, and there is no on-the-job search.

Given any distribution of posted wages $F(w)$, it should be obvious that each type of worker will have a reservation wage $w_{j}$ such that he accepts $w \geq w_{j}$ and rejects $w<w_{j}$, with $w_{j+1}>w_{j}$. It is equally apparent that, in any equilibrium, no firm would post anything other than one of the reservation wages, as a firm posting $w \in\left(w_{j}, w_{j+1}\right)$ could reduce $w$ down to $w_{j}$ and make more profit per worker without changing the set of workers who accept. A special case of this is the Diamond (1971) result when $K=1$ : with homogeneous workers say $L_{1}=1$, without loss of generality - all firms post $w_{1}$. Moreover, in this case $w_{1}=b_{1}$. To see why, assume all firms are posting $w>b_{1}$; then as long as $r>0$, a firm can post $w-\varepsilon$ for some $\varepsilon>0$ and still hire every worker it contacts. So in equilibrium, all firms must post $w=w_{1}=b_{1}$.

Consider the case $K=2$. Then there are at most two wages $w_{1}$ and $w_{2}$ posted in equilibrium. Let $\theta \in[0,1]$ be the fraction of firms posting $w_{2}$ and thus $1-\theta$ the fraction posting $w_{1}$. Let $U_{j}$ be the value function of an unemployed worker of type $j$ and $W_{j}(w)$ the value function of a type $j$ worker employed at $w$. Since we already know the only posted wages are $w_{1}$ and $w_{2}$, the relevant flow Bellman equations for unemployed workers are

$$
\begin{aligned}
& r U_{1}=b_{1}+\alpha_{w}(1-\theta)\left[W_{1}\left(w_{1}\right)-U_{1}\right]+\alpha_{w} \theta\left[W_{1}\left(w_{2}\right)-U_{1}\right] \\
& r U_{2}=b_{2}+\alpha_{w} \theta\left[W_{2}\left(w_{2}\right)-U_{2}\right]
\end{aligned}
$$


where we use the result that type 2 accepts $w_{2}$ but not $w_{1}$, while type 1 accepts both offers. Indeed, the reservation property implies $W_{1}\left(w_{1}\right)=U_{1}$ and $W_{2}\left(w_{2}\right)=U_{2}$, and so the expressions simplify to

$$
\begin{aligned}
& r U_{1}=b_{1}+\alpha_{w} \theta\left[W_{1}\left(w_{2}\right)-U_{1}\right] \\
& r U_{2}=b_{2} .
\end{aligned}
$$

Again using the reservation property, the employed workers’ Bellman equations are

$$
\begin{aligned}
& r W_{1}\left(w_{1}\right)=w_{1} \\
& r W_{1}\left(w_{2}\right)=w_{2}+\delta\left[U_{1}-W_{1}\left(w_{2}\right)\right] \\
& r W_{2}\left(w_{2}\right)=w_{2} .
\end{aligned}
$$

Taken together, these equations imply $w_{2}=b_{2}$ and

$$
w_{1}=\frac{(r+\delta) b_{1}+\alpha_{w} \theta b_{2}}{r+\delta+\alpha_{w} \theta} \text {. }
$$

Notice $w_{1}$ is a weighted average of $b_{1}$ and $b_{2}$, and $w_{1}>b_{1}$ if and only if $\theta>0$. Type 1 workers do not accept $w=b_{1}$ if $\theta>0$, because there is a chance of getting $w_{2}=b_{2}$. Notice $\partial w_{1} / \partial \theta>0$.

Now consider firms. For now we follow Burdett-Mortensen and assume each firm is interested in maximizing steady state profit. To compute this, let $\rho_{j}$ be the probability a random unemployed worker accepts $w_{j}$. Then a firm posting $w_{j}$ hires at rate $\alpha_{f} \rho_{j}$, the rate at which it meets workers times the probability they accept, and expects to earn $\left(y-w_{j}\right) /(r+\delta)$ from each worker it hires, where $\delta$ is an exogenous rate at which matches end. Hence, firms care about ${ }^{5}$

$$
\Pi_{j}=\frac{\alpha_{f} \rho_{j}\left(y-w_{j}\right)}{r+\delta} .
$$

For a firm posting $w_{2}, \rho_{2}=1$, and for a firm posting $w_{1}, \rho_{1}=L_{1} u_{1} /\left(L_{1} u_{1}+L_{2} u_{2}\right)$, where $u_{j}$ is the steady state unemployment rate for type $j$ workers, with $u_{1}=\delta /\left(\delta+\alpha_{w}\right)$ and

\footnotetext{
${ }^{5}$ The original Burdett-Mortensen model actually proceeds by noting that in steady state a firm posting $w_{j}$ ends up with a stock $\alpha_{f} \rho_{j} / \delta$ of workers and is interested in maximizing $\alpha_{f} \rho_{j} / \delta\left(y-w_{j}\right)$. This yields exactly the same results in the model under consideration.
} 
$u_{2}=\delta /\left(\delta+\alpha_{w} \theta\right)$. Hence,

$$
\rho_{1}=\frac{L_{1}\left(\alpha_{w} \theta+\delta\right)}{L_{1}\left(\alpha_{w} \theta+\delta\right)+L_{2}\left(\alpha_{w}+\delta\right)}
$$

We are interested in the sign of $\Pi_{2}-\Pi_{1}$, since this determines the optimal wage posting strategy. This is equal in sign to $y-w_{2}-\rho_{1}\left(y-w_{1}\right)$, which, after inserting $\rho_{1}, w_{1}$ and $w_{2}$ and simplifying, can be shown to be equal in sign to the following linear function of $\theta$ :

$$
\begin{aligned}
T(\theta)= & \left\{\left[L_{1}(r+\delta)+L_{2}\left(\alpha_{w}+\delta\right)\right]\left(y-b_{2}\right)-L_{1}(r+\delta)\left(y-b_{1}\right)\right\} \alpha_{w} \theta \\
& +\left[L_{1} \delta(r+\delta)+L_{2}\left(\alpha_{w}+\delta\right)(r+\delta)\right]\left(y-b_{2}\right) \\
& -L_{1} \delta(r+\delta)\left(y-b_{1}\right)
\end{aligned}
$$

The following best response condition must hold in any equilibrium:

$$
\theta=0 \text { if } T(0)<0 ; \theta=1 \text { if } T(1)>0 \text {; and if } \theta \in(0,1) \text { then } T(\theta)=0 \text {. }
$$

When $T(\theta)=0$, we can solve explicitly for

$$
\theta=\frac{r+\delta}{\alpha_{w}} \frac{L_{1} \delta\left(y-b_{1}\right)-\left[L_{1} \delta+L_{2}\left(\alpha_{w}+\delta\right)\right]\left(y-b_{2}\right)}{\left[L_{1}(r+\delta)+L_{2}\left(\alpha_{w}+\delta\right)\right]\left(y-b_{2}\right)-L_{1}(r+\delta)\left(y-b_{1}\right)}
$$

Proposition 1 For all $\alpha_{w}$, there exists a unique solution to (3), and $0<\theta<1$ iff $y<y<\bar{y}$ where

$$
\underline{y}=b_{2}+\frac{\delta L_{1}\left(b_{2}-b_{1}\right)}{\left(\alpha_{w}+\delta\right) L_{2}} \text { and } \bar{y}=\underline{y}+\frac{r \alpha_{w} L_{1}\left(b_{2}-b_{1}\right)}{\left(r+\alpha_{w}+\delta\right)\left(\alpha_{w}+\delta\right) L_{2}} .
$$

Proof. Existence is easy. If $T(0) \leq 0$ then $\theta=0$ satisfies (3). Suppose $T(0)>0$. If $T(1) \geq 0$ then $\theta=1$ satisfies (3). If $T(1)<0$ then by continuity there exists a $\theta \in(0,1)$ that satisfies (3). Uniqueness follows from the result that $T^{\prime}(\theta)<0$ at any $\theta$ such that $T(\theta)=0$, which is easily verified to be true as long as $y \in(y, \bar{y})$. The thresholds $\underline{y}$ and $\bar{y}$ come from checking when $T(0)>0$ and $T(1)<0$.

When productivity is low, all firms pay $w_{1}=b_{1}$, when it is high, all firms pay $w_{2}=b_{2}$, and when it is in the intermediate region $(\underline{y}, \bar{y})$, there is wage dispersion. We can now solve explicitly for $w_{1}$ as well as the number of workers earning $w_{j}$, the unemployment rate, and so on, in the range where $\theta \in(0,1)$ by inserting (4) into (1). For example, normalizing $b_{1}=0$ without loss of generality, we have: 


$$
w_{1}=\frac{L_{2}\left(\delta+\alpha_{w}\right)\left(y-b_{2}\right)-L_{1} \delta b_{2}}{r L_{1}} .
$$

Notice $w_{1}=b_{1}=0$ at $y=\underline{y}$ and $w_{1}$ is (linearly) increasing in $y$ up to $w_{1}=\frac{\alpha_{w} b_{2}}{r+\delta+\alpha_{w}}$ at $y=\bar{y}$. The distribution of wages paid can be calculated easily given the steady-state conditions: $w=w_{1}$ with probability $1-\pi$ and $w=w_{2}$ with probability $\pi$, where $\pi=\frac{\ell_{2}}{\ell_{1}+\ell_{2}}$ and $\ell_{i}$ is the steady-state measure of workers who are employed at $w_{i}$. This is to be contrasted with the distribution of wages posted, which is given by $w=w_{1}$ with probability $1-\theta$ and $w=w_{2}$ with probability $\theta$. Typically, $\pi$ is bigger than $\theta$ since $w_{2}$ firms have more workers than $w_{1}$ firms, which is precisely how they can have equal profits. Notice that in this model we can have a decreasing density, in the sense that $\pi<1 / 2<1-\pi$. This is in contrast to the basic Burdett-Mortensen model where the density is increasing, contrary to the data. Of course, in the $K=2$ case, our density is not very realistic in another sense - there are only two wages. We show below how to generalize this.

It remains to discuss the arrival rates. As we mentioned earlier, the measure of firms is fixed at unity and each firm will hire as many workers as it can get. Suppose we assume a CRS meeting technology $m\left(n_{u}, n_{f}\right)$, where $n_{u}$ is the number of unemployed workers and $n_{f}=1$ is the number of firms. Then the rate at which workers contact firms is $\alpha_{w}=m\left(n_{u}, n_{f}\right) / n_{u}$, which given $n_{f}=1$ and $n_{u}=L_{1} u_{1}+L_{2} u_{2}=L_{1} \frac{\delta}{\delta+\alpha_{w}}+L_{2} \frac{\delta}{\delta+\alpha_{w} \theta}$ can be written

$$
\alpha_{w}=\frac{m\left(L_{1} \frac{\delta}{\delta+\alpha_{w}}+L_{2} \frac{\delta}{\delta+\alpha_{w} \theta}, 1\right)}{L_{1} \frac{\delta}{\delta+\alpha_{w}}+L_{2} \frac{\delta}{\delta+\alpha_{w} \theta}} .
$$

An equilibrium is then a pair $\left(\alpha_{w}, \theta\right)$ satisfying equations (3) and (6). Once $\alpha_{w}$ is known, $\alpha_{f}=m\left(n_{u}, n_{f}\right) / n_{f}$ can be calculated, but notice that $\alpha_{f}$ only affects the level of profits and not the sign of $\Pi_{2}-\Pi_{1}$, and so it does not affect the equilibrium values of $\theta, w_{j}$ and so on.

Consider for the sake of illustration the special case where $m\left(n_{u}, n_{f}\right)=A \min \left\{n_{u}, n_{f}\right\}=$ $A \min \left\{L_{1} u_{1}+L_{2} u_{2}, 1\right\}$, a matching function that arises in various applications (see, e.g., Lagos, 2000). This implies $m\left(n_{u}, n_{f}\right)=A n_{u}$ and hence $\alpha_{w}=A$ as long as $L_{1} u_{1}+L_{2} u_{2} \leq 1$ which always holds if $L \leq 1$, an assumption we are free to make. In this case, the arrival rate for workers is essentially exogenous. Hence, equilibrium is completely characterized by (3), and everything to be said about it is contained in Proposition 1.

We will not dwell on existence or uniqueness/multiplicity in the case of a general matching technology, but instead we present another, less extreme, example. Consider the CobbDouglas specification $m\left(n_{u}, n_{f}\right)=A n_{u}^{1-\gamma} n_{f}^{\gamma}$. We can solve (6) in this case for 
Figure 1. Multiple Equilibria

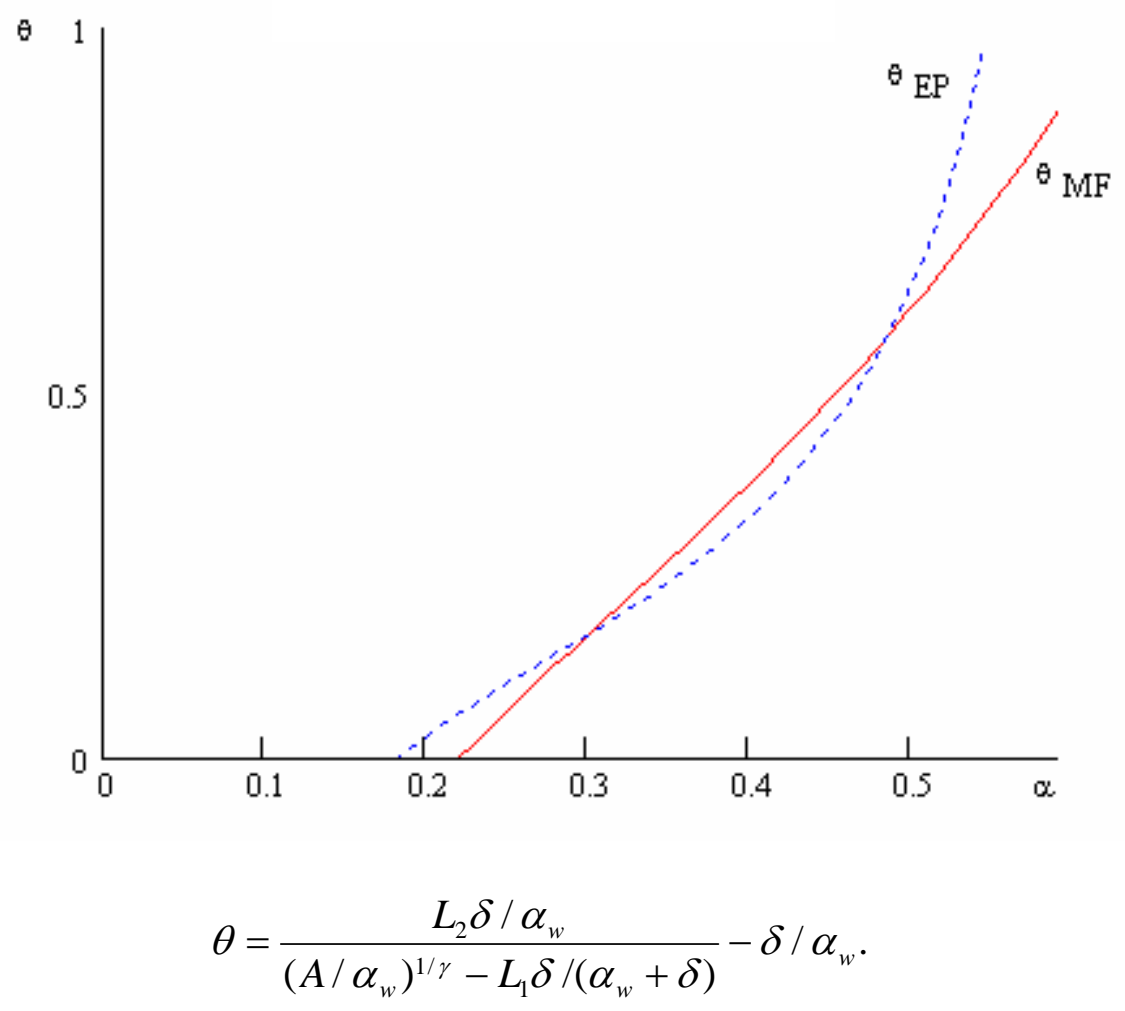

Figure 1 plots (4) and (7) in $\left(\alpha_{w}, \theta\right)$ space, the former labeled $\theta_{E P}$ for equal profit and the latter labeled $\theta_{M F}$ for matching function. As one can see, there are two solutions for $\theta \in(0,1)$.

Hence, the model not only is capable of generating wage dispersion, it also yields multiple equilibria with wage dispersion. ${ }^{6}$ The intuition for this result, which seems novel compared to the existing literature on multiplicity, is as follows. Suppose many firms are paying the high wage $w_{2}=b_{2}$, so that $\theta$ is relatively big. Then from (1) we see that $w_{1}$ is relatively big. This makes it relatively less profitable to try to get away with paying the low wage $w_{1}$ and hence more firms end up posting $w_{2}$.

\section{B. Alternative Assumptions}

An alternative assumption about firm behavior is that each employer may post at most one vacancy, along the lines of the models in Pissarides (2000), at cost $k$. Then firms maximize

\footnotetext{
${ }^{6}$ It is not easy to construct examples for realistic parameter values with $\theta \in(0,1)$ because, as one can see from (5), the interval $(y, \bar{y})$ is small when $r$ is small. The example in the figure uses $r=0.1, b_{1}=0.1, b_{2}=1.8, y=2.165, L_{1}=L_{2}=0.5, \delta=0.05, A=0.17$, and $\gamma=0.5$.
} 
the present discounted value of vacancies (as opposed to steady state profit). Mortensen (2000) shows that adopting this alternative scenario gives similar results in the basic BurdettMortensen model, under some conditions, and we want to see how it affects outcomes here.

Let $V_{i}$ denote the value of a firm with a vacancy posting wage $w_{i}$, and $J_{i}$ the value of having the job filled. Then we have

$$
\begin{aligned}
& r V_{j}=\alpha_{f} \rho_{j}\left(J_{j}-V_{j}\right)-k \\
& r J_{j}=y-w_{j}+\delta\left(V_{j}-J_{j}\right) .
\end{aligned}
$$

Solving the system yields

$$
r V_{j}=\frac{\alpha_{f} \rho_{j}\left(y-w_{j}+k\right)}{r+\delta+\alpha_{f} \rho_{j}}-k
$$

Compared with $\Pi_{j}$, the differences are that $k$ appears, and that $\alpha_{f} \rho_{j}$ shows up in the denominator of $V_{j}$. Inserting $\rho_{1}$ and $w_{1}$ into $V_{2}-V_{1}$, we see that it takes the same sign as

$$
\begin{aligned}
T(\theta)= & \left\{\left[L_{1}+\frac{L_{2}\left(\delta+\alpha_{w}\right)}{r+\delta+\alpha_{f}}\right]\left(y-b_{2}\right)-L_{1}\left(y-b_{1}\right)+\frac{L_{2}\left(\delta+\alpha_{w}\right)}{r+\delta+\alpha_{f}} k\right\} \alpha_{w} \theta \\
& +\left[L_{1} \delta+\frac{L_{2}\left(\delta+\alpha_{w}\right)(r+\delta)}{r+\delta+\alpha_{f}}\right]\left(y-b_{2}\right)-L_{1} \delta\left(y-b_{1}\right) \\
& +\frac{L_{2}\left(\delta+\alpha_{w}\right)(r+\delta)}{r+\delta+\alpha_{f}} k .
\end{aligned}
$$

Assume first $k=0$. As in the previous model, the best response condition (3) must hold in any equilibrium, and for any $\alpha_{w}$ and $\alpha_{f}$, there exists a unique solution to this condition, with $0<\theta<1$ iff $\underline{y}<y<\bar{y}$ (although the values for $\theta$ and the thresholds $\underline{y}$ and $\bar{y}$ are different). Again we can use

$$
\alpha_{w}=\frac{m\left(L_{1} \frac{\delta}{\delta+\alpha_{w}}+L_{2} \frac{\delta}{\delta+\alpha_{w} \theta}, v\right)}{L_{1} \frac{\delta}{\delta+\alpha_{w}}+L_{2} \frac{\delta}{\delta+\alpha_{w} \theta}}
$$

to determine $\alpha_{w}$ and then $\alpha_{f}$, except here we need to replace $v=1$ with $v=$ $1-L\left(1-L_{1} u_{1}-L_{2} u_{2}\right)$ since, with $k=0$, all firms that do not have a worker are recruiting - in the previous model all firms were recruiting, even those that had workers, because there firms want to employ as many people as they can get.

Because now both $\alpha_{w}$ and $\alpha_{f}$ enter the $T$ function, we cannot use $m\left(n_{u}, n_{f}\right)=A \min \left\{n_{u}, n_{f}\right\}$ 
to eliminate the arrival rates from $T$, as we did in the previous model: we can eliminate $\alpha_{w}=A$, but that still leaves $\alpha_{f}$. However, a different trick to simplify matters is to assume equal numbers of workers and firms: $L=1$. Then, given we are assuming $k=0$ so that all firms post vacancies, every filled job takes one worker and one vacancy off the market, leaving the ratio $n_{u} / n_{f}$ unchanged. Hence, constant returns in the matching function implies the arrival rates are again effectively exogenous, and all that one needs to determine is $\theta{ }^{7}$ Now consider $k>0$, so not all firms necessarily post vacancies. Free entry implies $V_{j}=0$ for any $w_{j}$ that is actually posted. To focus on the more interesting outcomes, consider any equilibrium with $\theta>0$. Then some firms post $w_{2}=b_{2}$, so $V_{2}=0$ and we can solve (10) with $j=2$ for

$$
\alpha_{f}=\frac{k(r+\delta)}{y-b_{2}}
$$

This pins down $\alpha_{f}$, from which we can determine the vacancy-unemployment ratio $u / v$ through $\alpha_{f}=m(u / v, 1)$, and then $\alpha_{w}=m(1, v / u)$. Substituting $\alpha_{f}$ and $\alpha_{w}$ into $T$ then allows us to determine $\theta$, which completes the description of equilibrium.

\section{Discussion}

We have illustrated under various assumptions that simple models with ex ante heterogeneous workers can generate wage dispersion. As we said above, this is very much in the spirit of the Albrecht-Axell model, although the details of our set up are quite different. Moreover, this framework generalizes quite easily to the case of $K>2$ types. There will be $K$ reservation wages $w_{1}, \ldots, w_{K}$, and in equilibrium these are posted with probabilities $\theta_{1}, \ldots, \theta_{K}$ where $\sum_{j=1}^{K} \theta_{j}=1$. Of course, some values of $\theta_{j}$ may be 0 in equilibrium, but clearly no firm will post anything other than one of the $K$ reservation wages. Eckstein and Wolpin (1990) analyze this version of the model empirically.

However, this class of models, with any value of $K$, has a problem: the equilibrium is not robust to the introduction of any search cost $\varepsilon>0$. In the case of $K=2$, the high reservation wage workers (those with $b=b_{2}$ ) get zero surplus from search - they reject $w_{1}$, and while they may accept $w_{2}$, for them it is no better than unemployment. Hence they will not search if $\varepsilon>0$. But then no firm will post anything other than $w_{1}$ and we are back to Diamond (1971).

Obviously this is true for any $K$ : worker types with the highest $b_{K}$ get no surplus, so they

\footnotetext{
${ }^{7}$ It is not that we think that these special assumptions about matching are particularly good; we are simply providing specifications where the model generates simple results about the wage distribution without having to worry about arrival rates.
} 
drop out if $\varepsilon>0$, and there are $K-1$ types left, and so on. We cannot get robust wage dispersion with ex ante heterogeneity. Indeed, things are worse than one might think: once all but type 1 workers drop out, given $\varepsilon>0$, the type 1 workers will drop out as well and the market shuts down. Based on these observations, we think it is worth considering some alternatives to ex ante heterogeneity.

\section{Ex Post Heterogeneity}

\section{A. Permanent Shocks}

Consider a model where workers are ex ante homogeneous, but matches are ex post heterogeneous. In particular, when a worker contacts a firm, he draws at random a matchspecific $c \in\left\{c_{1}, \ldots, c_{K}\right\}$, where $c$ is the per period cost to accepting the job. For example, $c$ could be the cost of commuting, working with people you may or may not like, etc. ${ }^{8}$ For now $c$ is permanent for the duration of the match (later we will also consider the case where workers draw a new $c$ each period). As in the previous section, we start with $K=2$ and consider $K>2$ below. Thus, $c=c_{1}$ with probability $\lambda$ and $c=c_{2}>c_{1}$ with probability $1-\lambda$. Assume $b+c_{2}<y$. Again, we begin by assuming that firms post wages to maximize steady state profit, as in Burdett-Mortensen.

It should be obvious that each worker now has two reservation wages: he accepts $w \geq w_{1}$ if he draws $c_{1}$ in a match, and accepts $w \geq w_{2}$ if he draws $c_{2}$. For the same reason as in the previous model, there will be at most two wages posted - no firm would post anything other than $w_{1}$ or $w_{2}$. We let $\theta$ be the fraction of firms posting $w_{2}$ as before, and we now let $W_{j}(w)$ be the value to having a job with wage $w$ and $c=c_{j}$ and $U$ the value of unemployed search. A key difference from the previous section is that here $U$ is not indexed by type - there are no types, as all workers are ex ante identical. Also, there $W_{j}(w)$ denoted the value function for a type $j$ worker employed at $w$, while here it is the value function for worker in a type $j$ match employed at $w$.

The reservation wage conditional on $c=c_{j}$ satisfies $W_{j}\left(w_{j}\right)=U$. The Bellman equation for unemployed workers is

$$
r U=b+\alpha_{w} \lambda \theta\left[W_{1}\left(w_{2}\right)-U\right]
$$

where we have used the facts that a worker who draws $c_{2}$ does not accept $w_{1}$, and that a worker who draws $c_{j}$ accepts $w_{j}$ but gets no surplus from doing so. Also,

\footnotetext{
${ }^{8}$ This model is similar to Burdett and Wright (1998), which is also based on non-pecuniary match-specific shocks, except there the wage is fixed. Burdett and Wright (1993) and Masters (1999) discuss ways to endogenize $w$ in these kinds of models, but they do not consider wage dispersion in their analyses.
} 


$$
r W_{j}(w)=w-c_{j}+\delta\left[U-W_{j}(w)\right]
$$

Solving these equations, we can derive

$$
\begin{aligned}
& w_{1}=b+c_{1}+\frac{\alpha_{w} \lambda \theta}{r+\delta}\left(c_{2}-c_{1}\right) \\
& w_{2}=b+c_{2}+\frac{\alpha_{w} \lambda \theta}{r+\delta}\left(c_{2}-c_{1}\right)
\end{aligned}
$$

A firm posting $w_{1}$ hires at rate $\alpha_{f} \lambda$ and expects to earn $\left(y-w_{1}\right) /(r+\delta)$ from each worker it hires. Similarly, a firm posting $w_{2}$ hires at rate $\alpha_{f}$ and expects to earn $\left(y-w_{2}\right) /(r+\delta)$. Therefore,

$$
\begin{aligned}
& \Pi_{1}=\frac{\alpha_{f} \lambda\left(y-w_{1}\right)}{r+\delta} \\
& \Pi_{2}=\frac{\alpha_{f}\left(y-w_{2}\right)}{r+\delta} .
\end{aligned}
$$

The same methods used above imply that $\Pi_{2}-\Pi_{1}$ takes the same sign as

$$
T(\theta)=(r+\delta)\left[(1-\lambda)(y-b)-\left(c_{2}-\lambda c_{1}\right)\right]-\alpha_{w} \lambda(1-\lambda)\left(c_{2}-c_{1}\right) \theta .
$$

An equilibrium still must satisfy the best response condition (3).

Proposition 2 For all $\alpha_{w}$, there exists a unique solution to (3), and $0<\theta<1$ iff $\underline{y}<y<\bar{y}$ where

$$
\underline{y}=b+\frac{c_{2}-\lambda c_{1}}{1-\lambda} \text { and } \bar{y}=\underline{y}+\frac{\alpha_{w} \lambda\left(c_{2}-c_{1}\right)}{r+\delta} .
$$

Proof. Existence is the same as in Proposition 1. Uniqueness is even easier here since $T^{\prime}(\theta)<0$ for all $\theta$. Again, the thresholds $y$ and $\bar{y}$ come from checking when $T(0)>0$ and $T(1)<0$.

One can again solve $T(\theta)=0$ for

$$
\theta=\frac{r+\delta}{\alpha_{w}} \frac{(1-\lambda)(y-b)-\left(c_{2}-\lambda c_{1}\right)}{\lambda(1-\lambda)\left(c_{2}-c_{1}\right)}
$$

Using this value of $\theta$, we can solve for wages in the case where $\theta \in(0,1)$ : 


$$
w_{j}=y+c_{j}-\frac{c_{2}-\lambda c_{1}}{1-\lambda} .
$$

When $\theta=0$, the unique posted wage is $w_{1}=b+c_{1}$, and when $\theta=1$, the unique posted wage is $w_{2}=b+c_{2}+\frac{\alpha_{w} \lambda}{r+\delta}\left(c_{2}-c_{1}\right)$. Note that $w_{2}>b+c_{2}$ because a worker who draws $c_{2}$ and has offer $w_{2}$ would prefer to turn it down and wait to get $c_{1}$ and $w_{2} \cdot{ }^{9}$

We can again consider different assumptions regarding firm behavior. When each firm can hire at most one worker and has to post a vacancy to recruit, the Bellman equations are again given by (8) and (9). If $k=0$, we have

$$
\alpha_{w}=m\left(1, \frac{1}{u\left(\theta, \alpha_{w}\right)}\right)
$$

where

$$
u\left(\theta, \alpha_{w}\right)=\frac{\delta}{\delta+\alpha_{w}[\theta+(1-\theta) \lambda]}
$$

is the unemployment rate. In an equilibrium with wage dispersion, the fraction $\theta$ of firms posting $w_{2}$ is still determined by (3) where $T$ is now given by (12). An equilibrium is a pair $\left(\theta, \alpha_{w}\right)$ satisfying (3) and (15). Again, in the special case $m(u, v)=A \min \{u, v\}$, we can guarantee $\alpha_{w}=A$ and equilibrium is fully characterized by (3).

If $k>0$, and assuming $\theta \in(0,1)$, the free entry condition $V_{j}=0$ pins down

$$
\alpha_{f} \rho_{j}=\frac{k(r+\delta)}{y-w_{j}}
$$

Given $\alpha_{f}$ we can determine $\alpha_{w}$ and this can be inserted into the $T(\theta)$ function, which then pins down $\theta$.

\section{B. The Law of Two Wages}

The model with ex post heterogeneity is not fragile with respect to introducing search costs. As long as $\theta>0$, it is clear that we can have $r U>b$, and hence workers would be willing to search even at a cost. It is also clear that we can generalize the analysis to the case where

\footnotetext{
${ }^{9}$ Also note that in this model $\bar{y}>\underline{y}$ even in the limit as $r \rightarrow 0$, unlike the model in the previous section where $\bar{y}=y$ at $r=0$. This makes it easier to construct relatively realistic examples with wage dispersion.
} 
$c=c_{1}, \ldots, c_{K}$ with probability $\lambda=\lambda_{1}, \ldots, \lambda_{K}$ and what we said goes through. In particular, there will exist $K$ conditional reservation wages $w_{1}, \ldots, w_{K}$ such that any worker accepts $w \geq w_{j}$ if he draws $c_{j}$ in a match. Things do not unravel here the way they did with ex ante heterogeneity because there are no types to drop out, and any worker gets positive gains from search as long as $\theta_{1}<1$, since then there is a chance he can get a job at a wage high enough to make him accept even if he draws $c_{j}>c_{1}$, but he gets lucky and draws $c=c_{1}$.

Now for something that may be more surprising (if one has not seen a version of it before). The usual Diamond logic guarantees that no firm will post any wage other than one of the $K$ reservation wages $w_{1}, \ldots, w_{K}$, so if we let $\theta_{j}$ be the fraction posting wage $w_{j}$, we know $\sum_{j} \theta_{j}=1$. This much is obvious. We now claim that generically there are never more than two wages actually posted. Adapting the language in Curtis and Wright (2004), we call this the law of two wages.

Proposition 3 For generic parameter values, we can have $\theta_{j}>0$ for at most two values of $j$. Proof. For any $K$, workers’ Bellman equations are

$$
\begin{aligned}
r U & =b+\alpha_{w} \sum_{j=1}^{K} \lambda_{j} \sum_{i=j}^{K} \theta_{i}\left[W_{j}\left(w_{i}\right)-U\right] \\
r W_{j}(w) & =w-c_{j}+\delta\left[U-W_{j}(w)\right] .
\end{aligned}
$$

The reservation property implies $W_{j}\left(w_{j}\right)=U$ for all $j$. This implies

$$
w_{j}=c_{j}+r U \text {. }
$$

Now consider profits and suppose $\theta_{i}>0, \theta_{j}>0, \theta_{k}>0$ for distinct $i, j$, and $k$. Then $\Pi_{i}=\Pi_{j}=\Pi_{k}=\max \left\{\Pi_{1}, \ldots, \Pi_{K}\right\}$. Using (16) we can write

$$
\begin{aligned}
\Pi_{j} & =\frac{\alpha_{f} \rho_{j}\left(y-w_{j}\right)}{r+\delta} \\
& =\frac{\alpha_{f} \rho_{j}\left(y-c_{j}-r U\right)}{r+\delta} \equiv g_{j}(U)
\end{aligned}
$$

where $\rho_{j}=\sum_{h=1}^{j} \lambda_{h}$ is the probability a random worker accepts $w_{j}$. Note that $g_{j}(U)$ depends only on $U, j$ and exogenous variables. The condition $\Pi_{i}=\Pi_{j}=\Pi_{k}$ therefore implies

$$
g_{i}(U)=g_{j}(U)=g_{k}(U) \text {, }
$$

a system of two equations in one unknown. For generic parameter values, there does not exist a solution. 
Figure 2. Profit as a Function of $w$

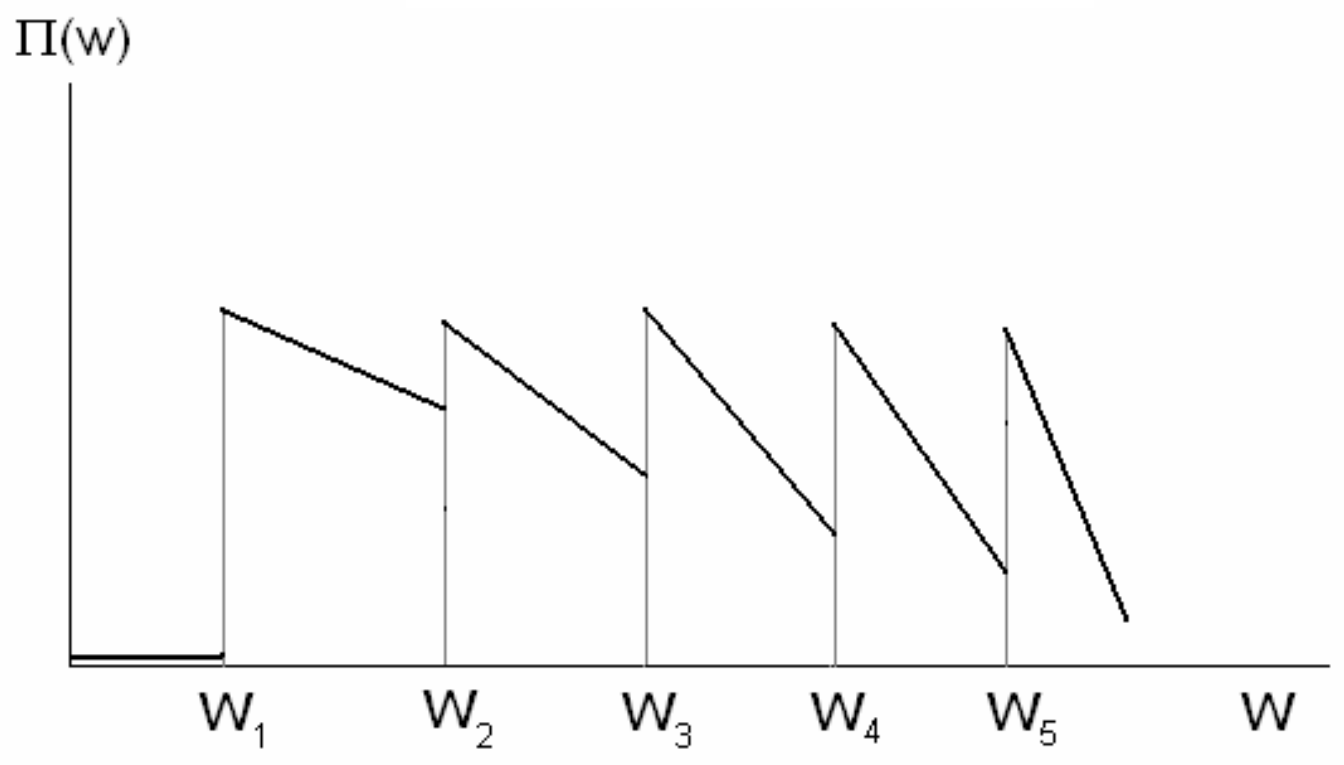

Although perhaps initially surprising, this result has a simple graphical representation. Figure 2 shows the steady state profit $\Pi(w)$ of a firm as a function of its posted wage. For $w<w_{1}$ the firm hires no one, so profit is 0 . At $w=w_{1}$, $(w)$ jumps up because now the firm hires any worker who shows up and draws $c=c_{1}$. For $w \in\left[w_{1}, w_{2}\right), \Pi(w)$ is linearly decreasing in $w$. And so on. ${ }^{10}$

If $w_{1}, \ldots, w_{K}$ were exogenous, then generically $\Pi(w)$ will attain a maximum at a unique $w_{j}$. But they are not exogenous. Hence, one might think they can adjust endogenously until there are multiple $w_{j}$ maximizing $\Pi(w)$. This is precisely what we did in the case $K=2$ to get $\Pi_{1}=\Pi_{2}$. However, the reservation wages are all related by (16); hence, it may be possible to adjust one of them so that $\Pi(w)$ is maximized at more than one point, but we cannot independently adjust another one so that $\Pi(w)$ is maximized at more than two points. ${ }^{11}$

\footnotetext{
${ }^{10}$ Notice the slopes of the decreasing segments are steeper for higher $w$, simply because higher wage firms have more workers.

11 Jafarey and Masters (2003) analyze a model related to Curtis-Wright, except the matchspecific shock is a uniformly distributed continuous variable. They show this implies a single price can be posted in equilibrium (this is not inconsistent with the law of two prices, of course, which says there are no more than two). Intuitively, with a continuum of shocks there is a continuum of reservation wages, and the function $\Pi(w)$ in Figure 2 does not have discrete jumps. Generalizing Jafarey and Masters, one can show there is a single wage posted if we assume a continuous distribution, say $G$, that satisfies $G^{\prime \prime} \geq-2 G^{\prime 2} /(1-G)$ (see Curtis
} 
A detail that may be pointed out is the following: when we say $g_{i}(U)=g_{j}(U)=g_{k}(U)$ constitutes two equations in one unknown, one might worry that in fact the $g$-functions depend on $\alpha_{f}$, which itself may be endogenous. If arrival rates are endogenous, however, then we need to add one more condition to determine them and hence we still have more equations than unknowns. More explicitly, consider the version of the model where each firm can hire at most one worker and has to post a vacancy to recruit (this also allows us to show the results hold in different versions of the model and do not depend on maximizing $\Pi(w)$, e.g. A firm’s Bellman equations are still given by (8) and (9).

The equal profit condition can be written $V_{i}\left(U, \alpha_{f}\right)=V_{j}\left(U, \alpha_{f}\right)=V_{k}\left(U, \alpha_{f}\right)$ where, using (16),

$$
V_{i}\left(U, \alpha_{f}\right)=\frac{\alpha_{f} \rho_{i}\left(y-c_{j}-r U+k\right)}{r+\delta+\alpha_{f} \rho_{i}}-k
$$

The free entry condition requires $V_{j}\left(U, \alpha_{f}\right)=0$, or

$$
\alpha_{f}=\frac{(r+\delta) k}{\rho_{i}\left(y-c_{j}-r U\right)}
$$

Substituting the equilibrium value of $\alpha_{f}$ into $V_{i}\left(U, \alpha_{f}\right)=V_{j}\left(U, \alpha_{f}\right)=V_{k}\left(U, \alpha_{f}\right)$, we again have a system of two equations in the one unknown $U$.

\section{OTHER MODELS}

\section{A. Transitory Shocks}

The above model assumes that when a worker and firm meet, the match-specific shock $c$ is kept forever. Suppose now that $c$ is an i.i.d. draw each period in a given match, after the job is accepted. Each period, workers decide whether to come to work or to stay home that day without losing the job. ${ }^{12}$ Consider the case where $K=2$, so $c=c_{1}$ with probability $\lambda$ and

and Wright, 2004, for details and other references). This is not a particularly general condition, however, and we should not expect a single wage to be typical - we already showed by example that it is easy to get two.

${ }^{12}$ We assume they do not lose their job if they stay home one day because a threat by firms to fire a worker for not showing up is not credible - the best they could do is replace him after some time with someone identical. This is similar to the efficiency wage model of Shapiro and Stiglitz (1984), e.g., in the sense that workers may shirk and firms may choose to pay them enough so they will not shirk, but in our model layoffs are not used as a discipline device. 
$c=c_{2}$ with probability $1-\lambda$. We continue to assume $b+c_{2}<y$. All workers have a common reservation wage for accepting a job, say $w_{1}$. At that wage they will come in only on days when $c=c_{1}$. However, a firm may choose to pay $w_{2}>w_{1}$ to entice workers to come in even on days when they draw $c=c_{2}$. Obviously, no firm would ever post anything other than $w_{1}$ or $w_{2}$, and as always we let $\theta$ be the fraction posting the latter. The Bellman equations for workers are

$$
\begin{aligned}
r U & =b+\alpha_{w} \theta\left[W\left(w_{2}\right)-U\right]+\alpha_{w}(1-\theta)\left[W\left(w_{1}\right)-U\right] \\
r W(w) & =E \max \{w-c, b\}+\delta[U-W(w)],
\end{aligned}
$$

where $E \max \{w-c, b\}$ reflects the fact that, at a given wage, the worker will stay home for realizations of $c$ above $w-b$.

It should be obvious that $w_{1}-c_{2}<b$ and $w_{2}-c_{2}=b$; hence $E \max \left\{w_{1}-c, b\right\}=$ $\lambda\left(w_{1}-c_{1}\right)+(1-\lambda) b$ and $E \max \left\{w_{2}-c, b\right\}=w_{2}-\left[\lambda c_{1}+(1-\lambda) c_{2}\right]$. The reservation property implies $W\left(w_{1}\right)=U$. Putting these facts together, we can solve for

$$
w_{1}=b+\frac{(r+\delta) c_{1}+\alpha_{w} \theta c_{2}}{r+\delta+\alpha_{w} \theta}
$$

Again notice that the reservation wage has to be more than enough to entice the worker to come in on his best day, as long as $\theta>0$, since the worker can always hold out for a job that pays enough to come in on a bad day, which delivers a positive surplus every time he has a good day with $c=c_{1}$.

For firms, we have

$$
\Pi_{j}=\frac{\alpha_{f} \rho_{j}\left(y-w_{j}\right)}{r+\delta},
$$

where $\rho_{j}$ now is the probability a worker shows up on any given day, given $w_{j}: \rho_{1}=\lambda$ and $\rho_{2}=1$. After inserting the wages, we can show that $\Pi_{2}-\Pi_{1}$ is equal in sign to

$$
T(\theta)=(r+\delta)\left[(1-\lambda)(y-b)-c_{2}+\lambda c_{1}\right]+(1-\lambda)\left(y-b-c_{2}\right) \alpha_{w} \theta
$$

Equilibrium still requires the best response condition (3). Notice that, in contrast to the other models, here we have $T^{\prime}>0$, and so there is the potential for multiple equilibria, even for a given $\alpha_{w}$.

Proposition 4 For all $\alpha_{w}$, we have the following: $\theta=0$ is the unique solution to (3) if 
$y<\underline{y} ; \theta=1$ is the unique solution if $y>\bar{y}$; and there are three solutions $\theta=0, \theta=1$ and $\theta \in(0,1)$ if $\underline{y}<y<\bar{y}$ where

$$
\underline{y}=b+c_{2}+\frac{(r+\delta) \lambda\left(c_{2}-c_{1}\right)}{\left(r+\delta+\alpha_{w}\right)(1-\lambda)} \text { and } \bar{y}=\underline{y}+\frac{\alpha_{w} \lambda\left(c_{2}-c_{1}\right)}{\left(r+\delta+\alpha_{w}\right)(1-\lambda)} .
$$

Proof. Similar to earlier results.

It is easy to generalize the analysis to any $K>2$ and to verify that the law of two wages holds. Suppose that more than one wage is posted in equilibrium. The lowest possible posted wage $w_{1}$ is the reservation wage. Every other posted $w_{j}$ will be equal to $c_{j}+b$ for some $j$. To see this, consider a firm posting $w \in\left(b+c_{j}, b+c_{j+1}\right)$. It would face the same probability of workers showing up on any given day by posting $b+c_{j}$. Hence, all wages posted must equal $b+c_{j}$ for some $j$, conditional on exceeding the reservation wage $w_{1}$. At the reservation wage $w_{1}$, however, this argument breaks down since if $w_{1}>b+c_{1}$ we cannot lower it and stay in business; as we saw above, with $K=2, w_{1}>b+c_{1}$ when $\theta>0$.

Having clarified the possible structure of equilibrium wages, consider the possibility that more than two are posted. Then $\Pi_{j}$ is still given by (17), but now $\rho_{j}=\sum_{h=1}^{j} \lambda_{h}$. The point is that $\Pi_{j}$ is a function of $w_{j}$ and exogenous parameters. Hence, we have the same problem as before: $\Pi_{i}=\Pi_{j}=\Pi_{k}$ constitutes two equations in one unknown. The law of two wages again holds for any $K$.

\section{B. The Crime Model}

We present one more model. To motivate this version, observe the following. In Sections 2 and 3, firms paying higher wages recruit at a faster rate. In Burdett-Mortensen, high wage firms recruit faster and additionally lose workers more slowly. Here, in the interest of completeness, we present a model where they lose workers more slowly only. Following Burdett, Lagos and Wright (2003), we interpret this as a model of crime. Thus, any employed worker randomly comes across an opportunity to commit crime at rate $\mu$, with gross reward $R$. There is a probability $v$ of getting caught, which means having to leave one's job and being forced into unemployment. More generally, in Burdett, Lagos and Wright, when a worker is caught he is put in jail for a while, which means he obviously cannot keep his job. For simplicity here we assume jail time is zero, but still assume that a worker who gets caught loses his job, since this is what matters for the purpose of generating wage dispersion. $^{13}$

${ }^{13}$ As we mentioned above, in some efficiency wage models firms are supposed to punish workers who get caught engaging in bad behavior by laying them off, even though they have incentive not to lay them off ex post. Having a third party (e.g., the police) take workers 
Workers are ex ante homogeneous, and have a common reservation wage $w_{0}$. Firms can hire any worker they contact by posting $w_{0}$. However, a plausible alternative is to pay a wage above $w_{0}$ to induce a worker to refrain from crime. Firms may find this profitable since, after all, they suffer a capital loss when workers leave. To see how it works, let $w_{1}>w_{0}$ denote the rime wage at which a worker would refrain from crime rather than risk losing his job, defined by $R+v\left[U-W\left(w_{1}\right)\right]=0$. It is clear that in equilibrium no firm would post anything other than $w_{0}$ or $w_{1}$. As above, let $\theta$ be the fraction of firms posting the higher wage.

The Bellman equations for workers are

$$
\begin{aligned}
r U & =b+\alpha_{w} \theta\left[W\left(w_{1}\right)-U\right] \\
r W\left(w_{0}\right) & =w_{0}+\mu R \\
r W\left(w_{1}\right) & =w_{1}+\delta\left[U-W\left(w_{1}\right)\right] .
\end{aligned}
$$

Although they accept $w_{0}$, they get no capital gain from doing so and suffer no capital loss from losing $w_{0}$. Using $R+v\left[U-W\left(w_{1}\right)\right]=0$ and $W\left(w_{0}\right)=U$, we can solve for

$$
\begin{aligned}
& w_{1}=b+(r+\delta+\alpha \theta) R / v \\
& w_{0}=b-\mu R+\alpha \theta R / v
\end{aligned}
$$

All firms recruit at the same rate $\alpha_{f}$, but those paying $w_{0}$ lose workers at rate $\delta+\mu v$ while those paying $w_{1}$ lose workers at rate $\delta$. Hence,

$$
\begin{aligned}
& \Pi_{0}=\frac{\alpha_{f}\left(y-w_{0}\right)}{r+\delta+\mu \nu} \\
& \Pi_{1}=\frac{\alpha_{f}\left(y-w_{1}\right)}{r+\delta} .
\end{aligned}
$$

Following the usual procedure, $\Pi_{1}-\Pi_{0}$ is proportional to

$$
T(\theta)=\mu v(y-b)-(r+\delta)^{2} R / v-\mu\left(2 r+2 \delta+\alpha_{w} \theta\right) R .
$$

For any $\alpha_{w}$, (3) is again an equilibrium condition. There is a unique solution to (3) and

away gets around this problem. Also note that for simplicity here we assume the unemployed do not engage in crime, but this is easily generalized. 


$$
\theta=\frac{\mu v(y-b)-(r+\delta)^{2} R / v+2 \mu(r+\delta) R}{\mu \alpha_{w} R}
$$

is in $(0,1)$ iff $y$ is in $(\underline{y,} \bar{y})$ where

$$
\begin{aligned}
& \underline{y}=b+\frac{(r+\delta)^{2} R / v+2 \mu(r+\delta) R}{\mu \nu} \\
& \bar{y}=\underline{y}+\alpha_{w} R / \mu .
\end{aligned}
$$

Generalizing this model, suppose crime opportunities have potentially $K$ different payoffs, $R=R_{j}$ with probability $\mu_{j}$ for $j=1, \ldots, K$. We can also allow the probability of getting caught and hence losing one's job $v_{j}$ vary with the opportunity. There will be $K$ critical wages at which workers are just indifferent for some $j, R_{j}=v_{j}\left[W\left(w_{j}\right)-U\right\rfloor$, as well as a reservation wage $W\left(w_{0}\right)=U$. We can reorder the labels so that $w_{1}<w_{2}<\ldots<w_{K}$, and without loss of generality we consider the case where $w_{0}<w_{1}$ (otherwise we can ignore opportunities for low $j$ ). The generalized worker payoffs are

$$
\begin{aligned}
r U & =b+\alpha_{w} \sum_{j=0}^{K} \theta_{j}\left[W\left(w_{j}\right)-U\right] \\
r W(w) & =w+\delta[U-W(w)]+\sum_{i=j+1}^{K} \mu_{i}\left\{R_{i}+v_{i}[U-W(w)]\right\} .
\end{aligned}
$$

Profit from posting any $w_{j}$ is

$$
\Pi_{j}=\frac{\alpha_{f}\left(y-w_{j}\right)}{r+\delta+\xi_{j}},
$$

where $\xi_{j}=\sum_{i=j+1}^{K} \mu_{i} v_{i}$ is the probability a worker is forced to leave. We can have $\Pi_{i}=\Pi_{j}$ for $i \neq j$ since the higher profit per worker that comes with a lower wage could be offset by a higher rate at which a firm loses workers, as we saw when $K=2$. But generically, there is no way to have $\Pi_{i}=\Pi_{j}=\Pi_{k}$ for distinct $i, j$ and $k$, since the crime wages $w_{i}, w_{j}$ and $w_{k}$ are related through $\left.R_{j}=v_{j} \mid W\left(w_{j}\right)-U\right]$. Hence the law of two wages also holds here.

\section{EX ANTE AND Ex Post Combined}

We now assume that there are match-specific shocks and that individuals differ permanently in their valuation of unemployment. Let $K_{1}$ denote the number of types $b_{i} \in\left\{b_{1}, \ldots, b_{K_{1}}\right\}$ and $K_{2}$ the number of match-specific shocks $c_{j} \in\left\{c_{1}, \ldots, c_{K_{2}}\right\}$, arranged such that $c_{i}<c_{i+1}$ and 
$b_{j}<b_{j+1}$. Also, $\sum_{i=1}^{K_{1}} \lambda_{i}^{b}=\sum_{j=1}^{K_{2}} \lambda_{j}^{c}=1$ where $\lambda_{i}^{b}$ is the fraction of individuals with $b=b_{i}$ and $\lambda_{j}^{c}$ is the probability that any individual draws $c=c_{j}$. By the usual argument, there will be at most $K=K_{1} K_{2}$ different reservation wages, and so at most $K$ different posted wages.

Let $U_{i}$ be the value function for an unemployed type $i$ worker and $W_{i j}(w)$ the value function for a type $i$ worker employed at $w$ with $c=c_{j}$. The Bellman equations are

$$
\begin{aligned}
r U_{i} & =b_{i}+\alpha_{w} \sum_{j=1}^{K_{2}} \lambda_{j}^{c} \sum_{k=1}^{K} \theta_{k} \max \left\{W_{i j}\left(w_{k}\right)-U_{i}, 0\right\} \\
r W_{i j}(w) & =w-c_{j}+\delta\left[U_{i}-W_{i j}(w)\right]
\end{aligned}
$$

All wages are set so as to satisfy

$$
W_{i j}\left(w_{i j}\right)-U_{i}=0
$$

for some pair $(i, j) .{ }^{14}$ Profit is given by

$$
\Pi_{i j}=\frac{\alpha_{f} \rho_{i j}\left(y-w_{i j}\right)}{r+\delta}
$$

where we now define $\rho_{i j}=\sum_{z, k \in \Phi_{i j}} \lambda_{z}^{b} \lambda_{k}^{c}$ and $\Phi_{i j}=\left\{z, k \mid W_{z k}\left(w_{i j}\right)-U_{z} \geq 0\right\}$. We can now prove the generalized law of two wages.

Proposition 5 For generic parameter values, we can have $\theta_{k}>0$ for at most $2 K_{1}$ values of $k$.

Proof. Fix the worker type $z$ and suppose that three of his reservation wages are posted, say $w_{z i}, w_{z j}$ and $w_{z k}$, where these each satisfy

$$
W_{z t}\left(w_{z t}\right)-U_{z}=0
$$

for $t=i, j, k$. Then $\Pi_{z i}=\Pi_{z j}=\Pi_{z k}$. The reservation property implies $w_{z j}=c_{j}+r U_{z}$ and so the equal profit condition implies

$$
g_{z i}\left(U_{z}\right)=g_{z j}\left(U_{z}\right)=g_{z k}\left(U_{z}\right)
$$

\footnotetext{
${ }^{14}$ The number of reservation wages could be strictly less than $K$, since it may be that $w_{i j}=w_{h k}$ for $(i, j) \neq(h, k)$, although this is a nongeneric case.
} 
where

$$
g_{z j}\left(U_{z}\right)=\rho_{z j}\left(y-c_{j}-r U_{z}\right) .
$$

Once again, this is a system of two equations in one unknown, $U_{z}$, which cannot generically be satisfied. Therefore at most two different wages are posted for any given $z$, and hence the maximum number is $2 K_{1}$.

Combining ex ante and ex post heterogeneity is interesting for the following reason. Having ex ante heterogeneity with $K_{1}$ types delivers a possibly rich wage distribution, but with only ex ante heterogeneity the equilibrium is not robust - it unravels with any $\varepsilon>0$ search costs. Having ex post heterogeneity is robust, but it delivers very limited dispersion because even with $K_{2}$ values of the shock, we can have at most two posted wages in equilibrium, by the law of two prices. Combining ex ante and ex post heterogeneity remedies the shortcomings of both models - the resulting equilibria are robust and the model can deliver more interesting distributions with up to $2 K_{1}$ wages. $^{15}$

\section{Conclusion}

We have studied some alternative models of the labor market in which the law of one price does not hold. With ex ante heterogeneity, which is an old trick used to generate wage dispersion, we pointed out that equilibrium is actually not robust to the introduction of positive search costs. With ex post heterogeneity, we showed equilibria are robust, but the resulting wage dispersion is repressed by the law of two prices. We demonstrated how a combination of the alternative models captures the best elements (or avoids the difficulties) of the two individual approaches. We also presented some other models and showed how one can adopt some different assumptions, say about firm behavior. We hope that this will lead to additional work on a wider variety of models of wage dispersion.

${ }^{15}$ It is worth elaborating on why equilibria with ex post heterogeneity are robust to positive search costs. In the model with only ex ante heterogeneity, one worker type (the one with the highest value of $b$ ) will always have zero surplus, so any search cost induces this worker to drop out. In the model with ex ante and ex post heterogeneity, there are possible outcomes where some types have zero surplus, so it is possible for some types to drop out, but there are also equilibria where all workers have a positive surplus. Indeed, even if all but one type drop out, the remaining workers still get positive surplus, and so the market will remain active, as long as $\theta>0$. 


\section{REFERENCES}

Albrecht, James, and Bo Axell, 1984, “An Equilibrium Model of Search Unemployment,” Journal of Political Economy, Vol. 92, pp. 824-40.

Burdett, Kenneth, and Kenneth L. Judd, 1983, “Equilibrium Price Dispersion,” Econometrica Vol. 51, pp. 955-70.

Burdett, Kenneth, and Dale T. Mortensen, 1998, “Wage Differentials, Employer Size, and Unemployment,” International Economic Review, Vol. 39, pp. 257-73.

Burdett, Kenneth, and Randall Wright, 1993, "Search, Matching and Unions," in Panel Data and Labor Market Dynamics, ed. by H. Bunzel, P. Jensen and N. Westergard-Nielsen, pp. 411-26 (Amsterdam: North-Holland).

_, and Randall Wright, 1998, “Two-Sided Search with Nontransferable Utility,” Review of Economic Dynamics, Vol. 1, pp. 220-45.

Burdett, Kenneth, Ricardo Lagos, and Randall Wright, 2003, "Crime, Inequality, and Unemployment,” American Economic Review, Vol. 93, pp. 1764-77.

Curtis, Elisabeth, and Randall Wright, 2004, "Price Setting, Price Dispersion, and the Value of Money: Or, the Law of Two Prices,” Journal of Monetary Economics, Vol. 51, pp. 1599-1621.

Diamond, Peter A., 1971, “A Model of Price Adjustment,” Journal of Economic Theory, Vol. 3, pp. 156-68.

Eckstein, Zvi, and Gerard van den Berg, 2005, "Empirical Labor Search: A Survey,” forthcoming in Journal of Econometrics.

Eckstein, Zvi, and Kenneth I. Wolpin, 1990, "Estimating a Market Equilibrium Search Model from Panel Data on Individuals,” Econometrica, Vol. 58, pp. 783-808.

Jafarey, Saquib, and Adrian Masters, 2003, “Output, Prices and the Velocity of Money in Search Equilibrium,” Journal of Money, Credit and Banking, Vol. 35, pp. 871-88.

Lagos, Ricardo, 2000, “An Alternative Approach to Search Frictions,” Journal of Political Economy, Vol. 108, pp. 851-73.

Mortensen, Dale T., 2003, Wage Dispersion (Cambridge, United Kingdom: Zeuthen Lecture Book Series).

- and Christopher A. Pissarides, 1994, "Job Creation and Job Destruction in the Theory of Unemployment,” Review of Economic Studies, Vol. 61, pp. 397-415. 
Pissarides, Christopher, 2000, Equilibrium Unemployment Theory, $2^{\text {nd }}$ edition (Cambridge, Massachusetts: MIT Press).

Postel-Vinay, Fabian, and Robin, Jean-Marc, 2002, "Equilibrium Wage Dispersion with Worker and Employer Heterogeneity,” Econometrica, Vol. 70, pp. 2295-2350.

Shapiro, Carl, and Joseph E. Stiglitz, 1984, "Equilibrium Unemployment as a Worker Discipline Device,” American Economic Review, Vol. 74, pp. 433-44.

van den Berg, Gerald J., and Gert Ridder, 1998, "An Empirical Equilibrium Search of the Labor Market,” Econometrica, Vol. 66, pp. 1183-1221. 